\section{Original Article}

Dr. Shamim Sultana

BDS, PhD

Assistant Professor \& Head, Dept. of Oral

Anatomy \& Physiology

Pioneer Dental College, Dhaka

Dr. Md Asafuzzoha

BDS, MS (Thesis)

Conservative Dentistry \& Endodontics BSMMU, Dhaka

Dr. Hasibul Hasan

BDS, MS

Assistant Professor

Dept. of Conservative Dentistry \& Endodontics

Pioneer Dental College, Dhaka

Dr. Md. Sajedul Asif Farzan

BDS, MPH

Assistant Professor

Dept of Children Preventive \& Community Dentistry Pioneer Dental College, Dhaka

Dr. Khairul Matin BDS, PhD

Cariology and Operative Dentistry Department of Restorative Sciences COE program for Frontier Research on Molecular Destruction and Reconstruction of

Tooth and Bone, Tokyo Medical and Dental University, Tokyo

\title{
Induction of White Spot Enamel Lesion by S. mutans Biofilm in an Artificial Mouth System and Quantification by QLF
}

\section{Abstract :}

Objective: Aspects of an Artificial Mouth System (AMS) to model the caries process in bovine enamel biofilm induced white spot enamel lesions (WSEL) were analyzed. Methods: Square shaped and polished bovine enamel slabs were covered with modeling wax and $2.5 \mathrm{~mm}$ diameter window was prepared to expose enamel surface at the center of each slab. Artificial biofilms were grown inside an AMS on the slabs using freshly cultured S. muatns (MT8148) in suspended in phosphate buffered saline (PBS), heart infusion (HI) with $1 \%$ sucrose and PBS for 20,30 and $40 \mathrm{hrs}$ at $37^{\circ} \mathrm{C}$. The severity of demineralization was quantified by Quantitative Light-induced Fluorescence (QLF, Inspektor, Netherlands). All experiments were repeated three times ( $\mathrm{n}=5$ for each group). Results: A circular WSEL could clearly be detected at the center of each slab by naked eyes, which was prominent after dehydration by mild air drying. QLF data showed that severity of demineralization occurred with the increase of time interval, after 40 hours both $\Delta \mathrm{F}(23+/-2.9)$ and $\Delta \mathrm{Q}(148.75+/-$ $26.78)$ were significantly more compared to $\Delta \mathrm{F}(10.14+/-2.3)$ and $\Delta \mathrm{Q}$ (47.63+/-30.67) of 20 hours Conclusion: An advanced in vitro model of biolfilm induced WSEL was developed that showed promises to be useful in studying enamel demineralization.

Key Words : QLF, White Spot Enamel Lesion (WSEL), biofilm, S. mutans,

Introduction:

When the $\mathrm{pH}$ of the oral cavity drops below 5.5 , the hydroxyapatite crystalline lattice is damaged and the tooth surface becomes rough [1]. Enamel demineralization and white lesions occur during and sometimes remain after orthodontic treatment [2]. Therefore, either due to some altered environmental factors in the oral cavity or as an adverse effect of some dental treatment primarily occurred damages to the enamel structure are described to be as white spot enamel lesions (WSEL); appear as white spots on tooth surface clinically. However, there is contradiction in defining WSEL and a standard classification for non-invasive treatment or restoration and prevention is yet to be established. In the late 19th century Magitot [3]. Miller [4]. proposed the concept of artificial mouth systems. The complexity of oral environment, and the ethical problems associated with in vivo studies of oral diseases such as caries and periodontal diseases in humans have inevitably led to the development of laboratory models, which simulate the oral environment in vitro. These model systems are given the epithet 'artificial mouth' (AM). During such experimental procedures, real-time growth and development of dental plaque/ biofilm can be observed with various microscopic tools or analyzed using microbiological, biochemical and molecular methods. A prospective WSEL model similar to that of clinically diagnosed one for multi purpose use including studying demineralization and remineralization in tooth surface needs to be introduced.

Therefore, in an aim to investigate aspects of an Artificial Mouth System (AMS) to model the caries process in bovine enamel biofilm induced WSEL were analyzed. 


\section{Material and Methods :}

Approximately $4 \times 4 \times 2 \mathrm{~mm} 3$ sized forty eight bovine enamel blocks were used in this study. Enamel surfaces were flattened and polished with 800, 1200, 1500 silicon carbide papers. Each enamel block surface was covered with $1.4 \mathrm{~mm}$ thick paraffin wax before making a $2.5 \mathrm{~mm}$ diameter whole at the center of the wax.

The enamel blocks were placed on a Teflon holder around a flat bulb pH electrode of the AMS by using red utility wax and keeping the surface of the slabs horizontal to the bulb surface. The Teflon holder bearing the slabs was set through the bottom opening of the chamber by a silicon plug. Pooled sterile saliva was then poured on the slabs and the electrode from above and incubated for $30 \mathrm{~min}$ in order to obtain a coat of salivary pellicle.

The chamber encircled by water jacket was sealed with another silicon plug fitted with five stainless steel tubes (21 gauge). As a result, the chamber itself served as an incubator with a $37{ }^{\circ} \mathrm{C}$ inner temperature. The other end of the five stainless steel tubes were connected with five silicon tubes passing through a PC operated pump and one of them was used to collect $\mathrm{S}$. mutans suspensions, two to collect $\mathrm{HI}$ and the remaining two to collect PBS from the prepared stock. All liquids were pumped into the chambers for 40 hours at $6 \mathrm{ml}$ per hour per tube so that they can continually drop onto the center of the specimen holder. All liquids form water domes and are mixed by the force of gravity exerted from the falling liquid drops on the holder and which become diffusely distributed over all of the specimens. When the liquid domes reach their maximum height the mixture of excess liquid falls off from the edges of the holders. Both of the chambers were simultaneously operated and the $\mathrm{pH}$ on the flat bulb electrode was recorded continuously. After 20, 30 and 40 hours each block with artificial biofilms were washed with PBS. After removal of biofilms the wax was removed with knife and finally washed three times with PBS and wiped to dry the surface.

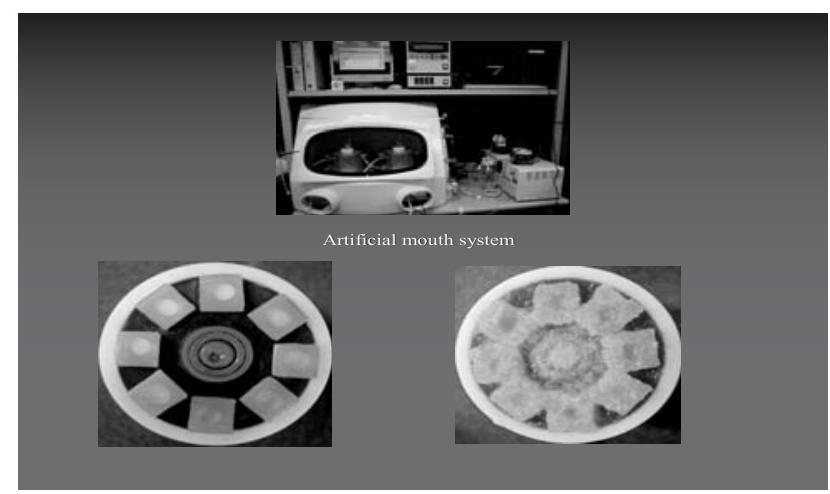

Fig 1: Artificial mouth system (top), samples assembled on to the Teflon holder (left) and formation of biofilms (right) after $20 \mathrm{hrs}$.

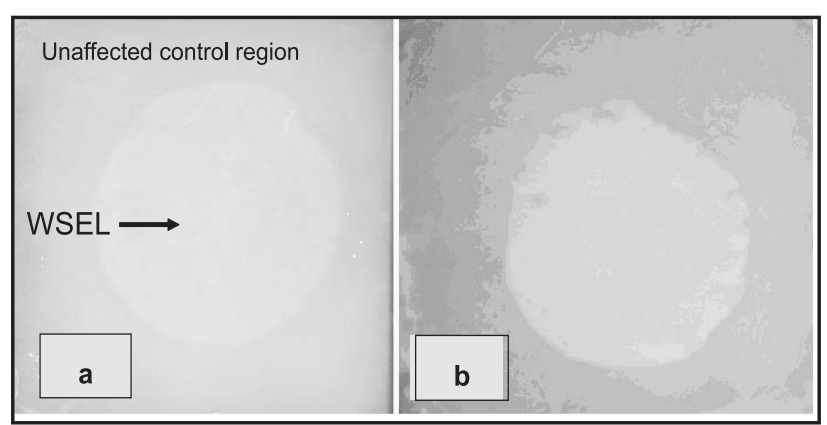

Fig 2: Digital photograph unaffected control region and WSEL $20 \mathrm{hrs}$ after biofilm formation [a] $40 \mathrm{hrs}$ after biofilm formation [b]

Then the specimens were visually examined and digital pictures were taken.

The severity of demineralization was quantified by QLF using the Inspektor? Pro software version 2.0.0.30 (Inspektor Dental Care BV, Amsterdam, Netherlands). QLF images and white-light digital photographs of each enamel block were taken at a fixed position in relation to the mounted camera. The part of the enamel surrounding the WSEL that remained intact considered as control region. Thereafter, during measuring the WSEL, part of the control region was included with demineralized region and data were recorded from $\Delta \mathrm{F}$ and $\Delta \mathrm{Q}$ values. $\Delta \mathrm{Q}$ value was recorded at the $5 \%$ threshold.

\section{Results :}

The $\mathrm{pH}$ beneath the biofilms a circular WSEL could clearly be detected at the center of each slab by naked eyes, which was prominent after dehydration by mild air drying (Fig 2a \& 2b).

QLF data showed that severity of demineralization occurred with the increase of time interval, after 40 hours both $\Delta \mathrm{F}(23+/-2.9)$ and $\Delta \mathrm{Q}(148.75+/-26.78)$ were significantly more compared to $\Delta \mathrm{F}(10.14+/-2.3)$ and $\Delta \mathrm{Q}(47.63+/-30.67)$ of 20 hours.

\section{Discussion :}

The present study, perhaps the first ever of this type, demonstrated induction of WSEL by biofilms grown in an AMS. The AMS used in this study to grow S. mutans biofilms is similar in principle to other biofilm reactors used previously for in vitro experiments. It is equipped with two chambers with a warm water jacket to maintain constant interior temperatures and also with a $\mathrm{N}_{2}(80 \%), \mathrm{H}_{2}(10 \%)$ and $\mathrm{CO}_{2}(10 \%)$ gas delivery unit to grow biofilms under anaerobic conditions. In addition, inside the chambers the liquid dome keeps the biofilm from direct contact with available $\mathrm{O}_{2}$. The reactor facilitates the continual monitoring of $\mathrm{pH}$ changes beneath the biofilm and in this study the reduction in $\mathrm{pH}$ was similar in all experiments; the $\mathrm{pH}$ began to fall from 7.35 within two hours and was reduced to below 4.0 by 20 hours. 
The teeth illuminator from an arc lamp using liquid light guide with a peak intensity of $370 \mathrm{~nm}$ was used. A yellow high pass filter $(520 \mathrm{~nm})$ is placed in front of a CCD microcamera which captures the tooth image. A live image of each enamel block under examination is displayed on a PC screen and when a quality image was visualized the operator captured that to save as video frame. The proprietary analysis software detects the darker areas of the image (video frame) and simulates the fluorescence radiance of sound enamel at the lesion site via a reconstruction algorithm. This is performed by a two dimensional liner interpolation of sound enamel values adjacent to the lesion.

The absolute decrease in fluorescence and is expressed in the value $\Delta \mathrm{F}$ (depth of the lesion). The program also calculates the area of lesion, in $\mathrm{mm} 2$ and from this can calculate $\Delta Q .5$

The rate of demineralization occurred with the increase of time intervals. Hardness measured by nanoindenter at the center of all lesions was significantly reduced from unaffected enamel, 40-hr sample showed about $98 \%$ reductions.

Perpendicularly trimmed surface topography after argon ion-etching by SEM clearly visualized depth of demineralized WSEL, widened inter-enamel prism channels, dislodgement of prism crystallites.

Biofilm induced WSEL surrounded by unaffected enamel showed uniformity which is important for quantification by QLF. The thickness of the paraffin wax allowed biofilms to retain inside the window without disturbance so uniform demineralization can continue.

\section{Conclusion:}

An advanced in vitro model of biolfilm induced WSEL was developed that showed promises to be useful in studying enamel demineralization employing QLF.

\section{References:}

1. Willmot DR. White lesions after orthodontic treatment: does low fluoride make a difference? J Orthod. 2004 Sep; 31(3):235-42; discussion 202.

2. Serra R, Otis LJ. Quantifying enamel luster, J Clin Dent. 2004; 15(3):83-7

3. Margitot E. Treatise on dental caries (Trans by Chandler TH). Boston: Osgood; 1878

4. Miller WD. The microorganisms of the human mouth. Philadelphi: white dental MFG: 1890

5. Hintze H, Wenzel A, Danielsen B, Nyvad B. Reliability of visual examination, fibre-optic transillumination, and bite-wing radiography, and reproducibility of direct visual examination following tooth separation for the identification of cavitated carious lesions in contacting approximal surfaces. Caries Res. 1998;32(3):204-9. 